\title{
Effect of Pressure on Boron Diffusion in Silicon
}

\section{Citation}

Zhao, Yuechao, Michael J. Aziz, Salman Mitha, and David Schiferl. 1997. Effect of pressure on Boron diffusion in Silicon. Materials Research Society Symposia Proceedings 442: 305-411.

\section{Published Version}

http://www.mrs.org/s_mrs/sec.asp?CID=1727\&DID=38980

\section{Permanent link}

http://nrs.harvard.edu/urn-3:HUL.InstRepos:2870608

\section{Terms of Use}

This article was downloaded from Harvard University's DASH repository, and is made available under the terms and conditions applicable to Other Posted Material, as set forth at http:// nrs.harvard.edu/urn-3:HUL.InstRepos:dash.current.terms-of-use\#LAA

\section{Share Your Story}

The Harvard community has made this article openly available.

Please share how this access benefits you. Submit a story.

Accessibility 


\title{
EFFECT OF PRESSURE ON BORON DIFFUSION IN SILICON
}

\author{
Yuechao Zhao ${ }^{1}$, Michael J. Aziz ${ }^{1}$, Salman Mitha ${ }^{2}$, and David Schiferl ${ }^{3}$ \\ ${ }^{1}$ Division of Engineering and Applied Sciences, Harvard University, Cambridge, MA 02138 \\ ${ }^{2}$ Charles Evans and Associates, Redwood City, CA 94063 \\ ${ }^{3}$ Los Alamos National Laboratory, Los Alamos, NM 87545
}

\begin{abstract}
We are studying the effect of pressure on boron diffusion in silicon in order to better understand the nature of the point defects responsible for diffusion. Si homoepitaxial layers deltadoped with boron were grown using molecular beam epitaxy. Diffusion anneals were performed in a high temperature diamond anvil cell using fluid argon as a pressure medium. Diffusivities were deduced from B concentration-depth profiles measured with using secondary ion mass spectrometry. Preliminary results indicate that pressure enhances B diffusion in $\mathrm{Si}$ at $850{ }^{\circ} \mathrm{C}$, characterized by an average activation volume of $-0.125 \pm 0.02$ times the atomic volume, and thus appear consistent with an interstitial-based diffusion mechanism. Results are compared with previous hydrostatic-pressure studies, with results in biaxially strained films, and with atomistic calculations of activation volumes for self diffusion.
\end{abstract}

\section{INTRODUCTION}

Because understanding and controlling diffusion related phenomena become increasingly important as semiconductor device dimensions decrease, diffusion in semiconductors has been heavily studied. Despite this emphasis there remains no consensus about the relative concentrations and mobilities of the point defects involved in the diffusion of many substitutional elements in $\mathrm{Si}$ [1]. A study of the dependence of the atomic diffusivity, D, on pressure, p, can provide valuable information to help elucidate the atomistic mechanism(s) of diffusion. The pressure-dependence of the diffusivity is characterized by the activation volume, $\Delta \mathrm{V}^{*}$ :

$$
-\mathrm{kT} \frac{\partial \ln \mathrm{D}(\mathrm{T}, \mathrm{p})}{\partial \mathrm{p}} \equiv \Delta \mathrm{V}^{*},
$$

where $\mathrm{k}$ is the Boltzmann constant and $\mathrm{T}$ is the temperature. When experimental conditions are such that the point defect concentrations equilibrate rapidly with the free surface compared to the experimental time scale then $\Delta \mathrm{V}^{*}$ is the sum of the formation volume $\Delta \mathrm{V}^{\mathrm{f}}$ and the migration volume $\Delta \mathrm{Vm}$. The formation volume is the volume change in the system upon formation, from the free surface, of a defect in its standard state, and the migration volume is the additional volume change when the defect reaches the saddle point in its migration path. $\Delta \mathrm{V}^{\mathrm{f}}$ characterizes the pressure-dependence of the equilibrium point defect concentration and $\Delta \mathrm{Vm}$ characterizes the pressure-dependence of the point defect mobility. Here we report preliminary results for the diffusion of boron, the technologically most important p-type dopant, in silicon. Effects associated with transient enhanced diffusion were avoiding by fabricating the sample without ion implantation. Boron delta-doped samples were grown by molecular beam epitaxy (MBE), which possess many advantages in diffusion studies [2].

There has been much recent work on diffusion of boron and other species under the biaxial strain conditions characteristic of strained-layer epitaxy. We compare these to our results and to those of molecular statics calculations using a thermodynamic formalism of Aziz [3] that relates diffusion under hydrostatic and nonhydrostatic stress states. When stresses become nonhydrostatic, the activation volume becomes the activation strain tensor: upon point defect formation and migration, dimension changes parallel and perpendicular to the direction of mass transport couple to different elements of the stress tensor. For diffusion normal to the surface of an (001) biaxially strained thin film with a diamond cubic structure, the formalism provides a 
relation between the activation volume obtained under hydrostatic conditions and the derivative, Q', of the apparent activation energy on biaxial strain:

$$
\Delta \mathrm{V}^{*}+\frac{3}{2} \frac{\mathrm{Q}^{\prime}}{\mathrm{Y}}= \pm \Omega+\left(\Delta \mathrm{V}_{/ /}^{\mathrm{m}}-\Delta \mathrm{V}_{\perp}^{\mathrm{m}}\right)
$$

where $\Delta \mathrm{V}_{/ /}^{\mathrm{m}}-\Delta \mathrm{V}_{\perp}^{\mathrm{m}}$ is the anisotropy in the migration volume, $\mathrm{Y}$ is the ratio of Young's modulus to one minus Poisson's ratio in the film, and the + and - signs are for vacancy and interstitial-based mechanisms, respectively. Deviations from eq. (2) will be observed if some of the point defect equilibration occurs at threading dislocations in biaxially strained films.

\section{EXPERIMENT}

The boron-doped Si samples used in the present study contain four boron spikes, grown by low temperature MBE [4], with a spacing of $70 \mathrm{~nm}$ and the closest B spike being $110 \mathrm{~nm}$ from the surface. The peak concentration of the spikes is about $10^{19}$ atoms $/ \mathrm{cm}^{3}$. Diffusion anneals at 850 ${ }^{\circ} \mathrm{C}$ were performed in a high temperature diamond anvil cell (DAC) [5] using fluid argon, cryogenically condensed from an argon gas of nominal purity $99.95 \%$, as an inert and hydrostatic pressure medium. The DAC was heated externally in a furnace as shown in Fig. 1. After the DAC was mounted into the furnace, the furnace was evacuated with a mechanical pump, the temperature was raised to $250-300^{\circ} \mathrm{C}$, and several cycles of flushing and evacuating with helium of nominal purity $99.999 \%$ were performed. Diffusion temperatures were reached after filling the furnace

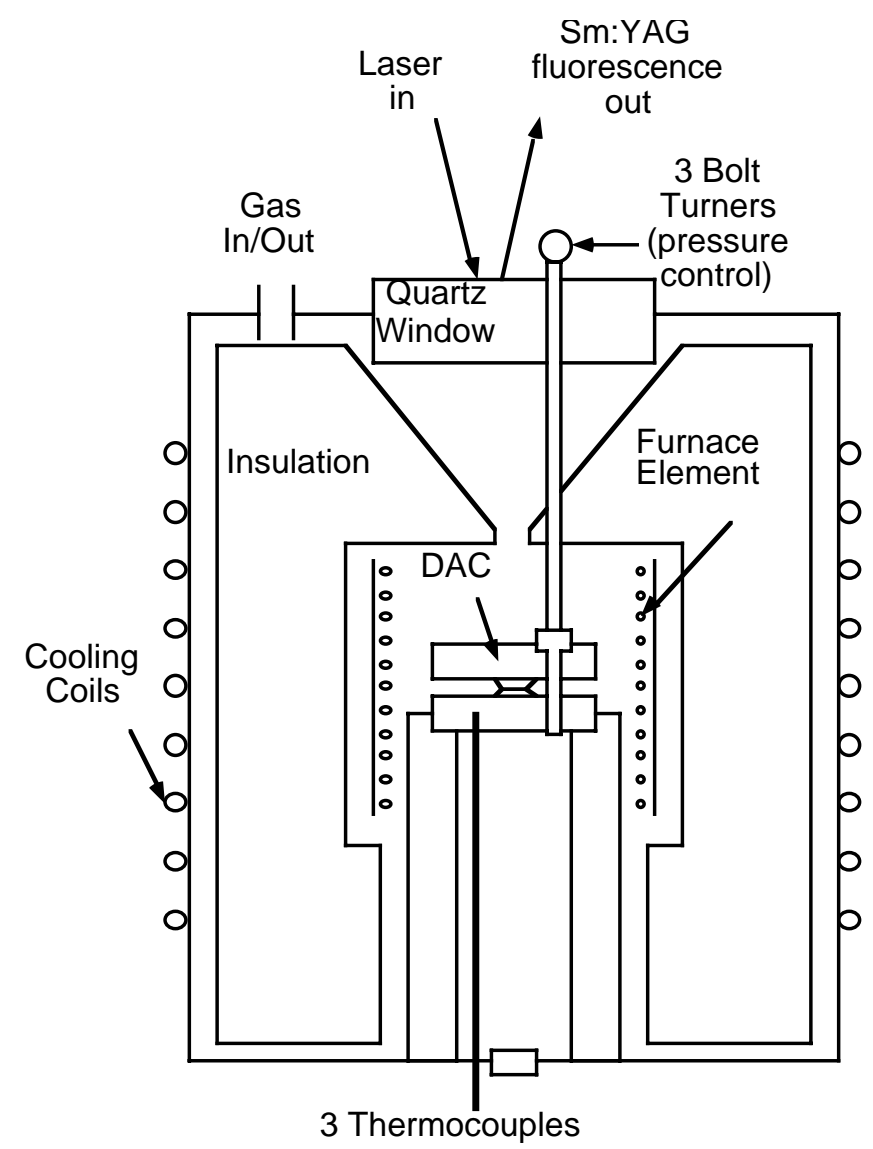

Figure 1. Schematic of high temperature-high pressure system used for pressure anneals. with helium at an overpressure of roughly 260 torr above atmospheric. The He ambient provides a uniform temperature field around the DAC and minimizes oxygen leakage into the furnace. Atmospheric-pressure anneals were performed with the DAC loaded with $1 \mathrm{~atm}$ of room-temperature Ar gas and sealed initially. At high temperatures, however, the DAC may open up, so that the furnace ambient may be in contact with the sample.

True anneal durations were about 2500 seconds. Temperatures were recorded using thermocouples embedded in the lower and upper plates supporting the diamond anvils and in the gap between the plates. These thermocouple readings never disagreed by more than $7{ }^{\circ} \mathrm{C}$. For the calculation of the diffusivity we used effective anneal times at $850{ }^{\circ} \mathrm{C}$, which were calculated using thermal histories recorded by a computer and an activation energy of $3.7 \mathrm{eV}$. Pressure can be adjusted in situ and was determined using the pressuredependence of the wavelength of Smdoped YAG fluorescence peaks Y1 through Y10 [6,7]. The uncertainty in the pressure determination is estimated to be $\pm 0.3 \mathrm{GPa}$.

Concentration-depth profiles of 
boron were measured using secondary ion mass spectrometry (SIMS) with an $8 \mathrm{keV} \mathrm{O}_{2}^{+}$primary ion beam. A linear sputter time-depth scale conversion for each profile was established using a linear regression to superpose the measured peak positions on the published peak positions established by profilometry [4]. The diffusivities of B were deduced from the profiles using Wu's $\sigma^{2}$-analysis [8], which relates the time-dependence of the standard deviation of a profile of any shape (we performed a separate analysis for each doping spike) to the diffusivity, assuming that the diffusivity is independent of concentration. Although it may affect the absolute magnitude of the calculated diffusivities, the assumption of concentration-independence should not significantly affect the measured activation volume. The use of a lower energy primary ion beam in order to improve the depth resolution was precluded by the requirement of a tight focus on samples with a typical size of $150 \times 150 \times 40 \mu \mathrm{m}^{3}$, defined by the size of the sample chamber of the DAC.

Fortunately, the diffusivities extracted from the $\sigma^{2}$ analysis are independent of instrumental resolution.

\section{RESULTS AND DISCUSSION}

Fig. 2 shows the B concentration-depth profiles in an as grown sample and a sample annealed at $3.5 \mathrm{GPa}$ for $2496 \mathrm{~s}$. Fig. 3 shows the preliminary results for the pressure-dependence of the diffusivity. Note that the closer to the surface the spikes are, the larger their diffusivities are. An argon anneal at Bell Labs [9] indicates that depth-dependence of the diffusivity appears to be a characteristic of this sample rather than of the atmosphere in this high-pressure study. However, the anomalously high diffusivity of the first spike in the 0-GPa sample, which may be related to the imperfect sealing by the DAC, has been discarded in the calculation of the apparent activation volume. Fig. 3 indicates that pressure apparently enhances B diffusion in $\mathrm{Si}$ at $850{ }^{\circ} \mathrm{C}$, characterized by an average activation volume of $-0.125 \pm 0.02 \Omega$. The values for diffusivities of $B$ obtained in the present study are systematically lower than the literature value of $\mathrm{B}$

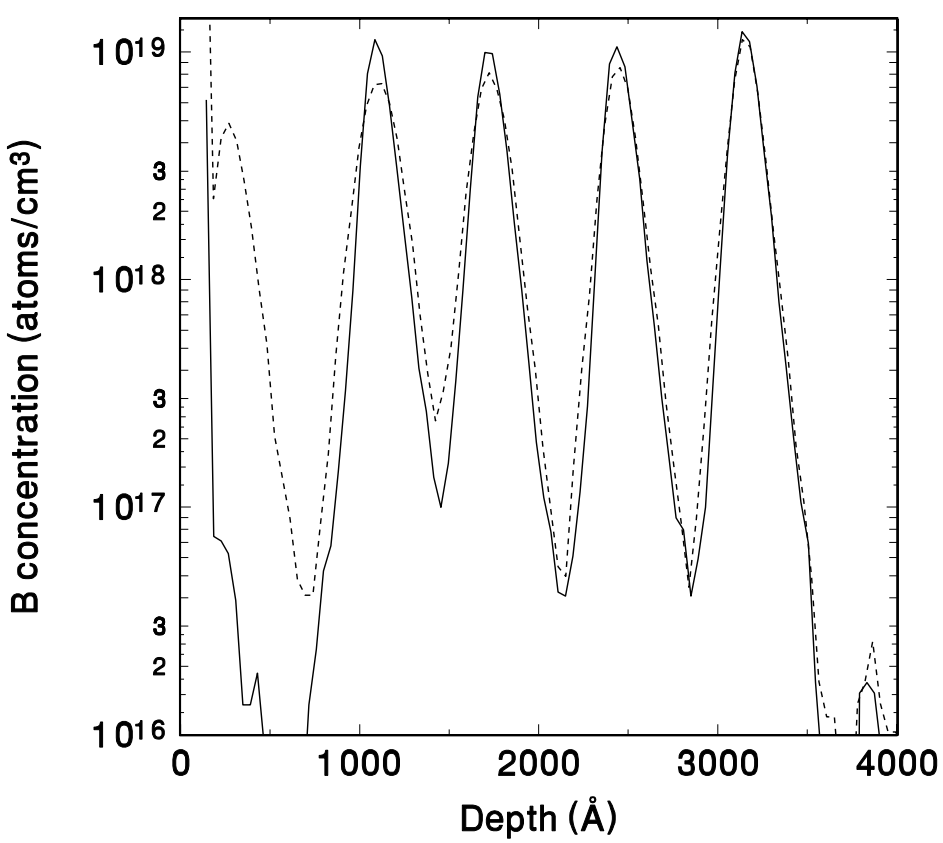

Figure 2. Typical concentration-depth profiles for as grown (solid line) and annealed (dashed line) samples. diffusivity in Si for $850{ }^{\circ} \mathrm{C}$ [10]. Instead, the diffusivity determined for the deepest spike corresponds to the literature value for about $780{ }^{\circ} \mathrm{C}$. This inconsistency remains a puzzle because the thermocouples in the DAC reproduced the melting point of $99.999 \%$ pure $\mathrm{Ag}(960$ $\left.{ }^{\circ} \mathrm{C}\right)$ within $10^{\circ}$. Although we have no information that the origin of this apparent inconsistency is independent of pressure, we will assume that it is pressureindependent for the purposes of the discussion below.

Our apparent activation volume differs from that of Södervall et al. [11], who reported $\Delta \mathrm{V}^{*}=(+0.27 \pm$ $0.25) \Omega$ over $1050-1230^{\circ} \mathrm{C}$. However, because their 


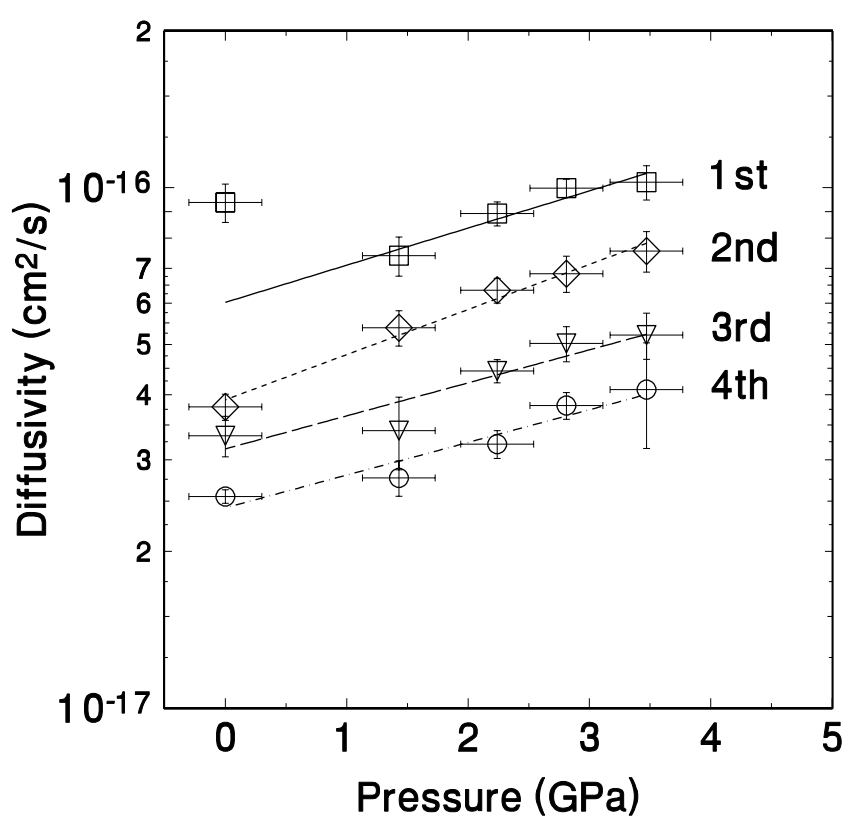

Figure 3. Preliminary results of pressure-dependence of $B$ diffusivities in Si measured in the present study. The spikes are labeled starting from that closest to the sample surface. samples were fabricated by ion implantation of boron, their results may be complicated by transient enhanced diffusion.

Our results are interpretable directly in terms of defect formation and migration volumes only if experimental conditions are such that the point defect concentrations equilibrate rapidly with the free surface compared to the experimental time scale. We have no proof that this is the case in the experiment reported here. If the process is nonequilibrium then this experiment may represent the effective pressure dependence of an interstitial injection process. Despite the obvious depth dependence of the diffusivity, which suggests that interstitial concentrations vary through the sample, we will make the openly naive assumption that there is rapid equilibration with the surface, in order to compare the observed behavior with the behavior that might be anticipated from the results of other experiments and models.

Qualitatively, the pressure enhancement is normally thought of as consistent with an interstitialbased mechanism. This is based on the common assumptions that the volume change upon relaxation around a point defect $\left(\Delta V^{\text {relax }}\right)$, as well as the migration volume, are substantially smaller than $\Omega$. When $\Delta \mathrm{V}^{\text {relax }}+\Delta \mathrm{V}^{\mathrm{m}}=0, \Delta \mathrm{V}^{*}=+1 \Omega$ for the vacancy mechanism and $-1 \Omega$ for interstitial-based mechanisms, exactly. It is now possible to be quantitative because $\Delta V^{r e l a x}$ and $\Delta \mathrm{Vm}^{\mathrm{m}}$ can be predicted using molecular dynamics or statics calculations. We know of no atomistic calculations of the volumetrics of boron diffusion in silicon. However, relaxation volumes have been calculated for self diffusion by interstitial-based mechanisms. Antonelli and Bernholc [12] used density functional theory with the local density approximation to calculate the volume of formation of the self-interstitial in the tetrahedral and bond-centered configurations. Tang et al. used the tight-binding approximation to calculate a formation volume of $-0.1 \Omega$ for the $<110>$ dumbbell self interstitial. If we assume that the formation and migration volumes for boron diffusion are close to those for self diffusion then we can at least see whether our results are reasonable.

Experimentally, Kuo et al. [13] isolated the effects of strain and composition on B diffusion in biaxially strained Si-Ge alloy thin films. They concluded that boron diffusion in silicon does not depend strongly on biaxial strain. Also, Cowern et al. [14] studied Si-Ge interdiffusion in biaxially strained multilayers under inert and oxidation-enhanced conditions and used a model to 


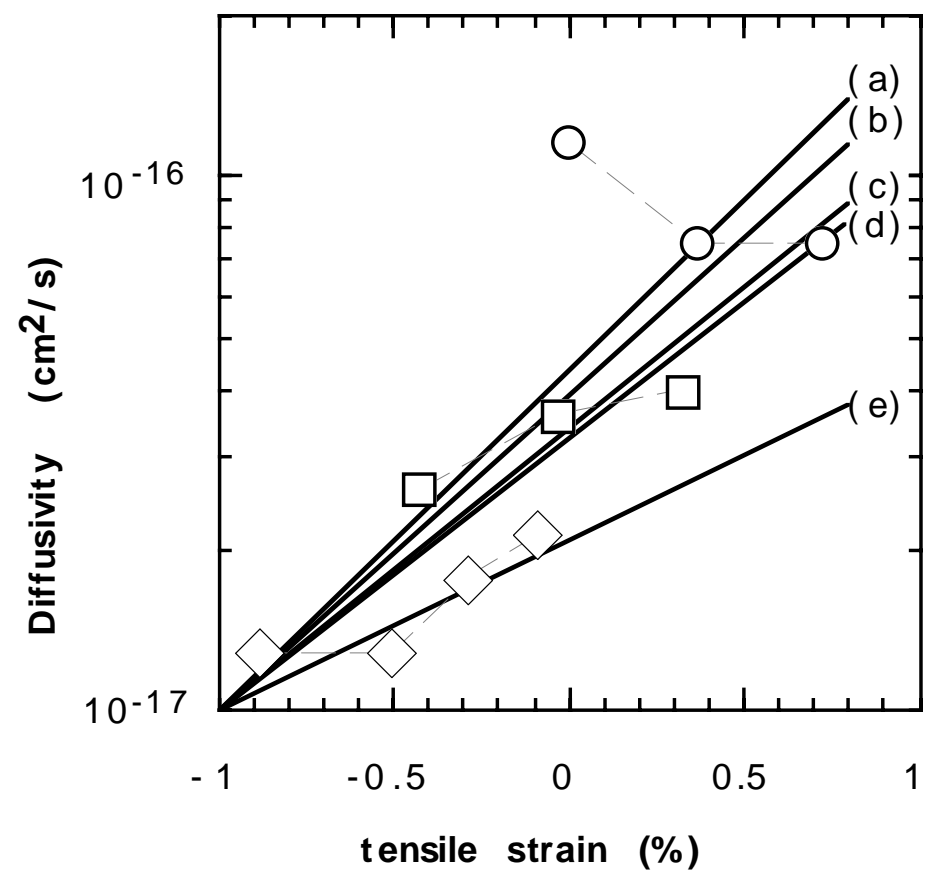

tensile strain (\%)
Figure 4. Preliminary comparison of interstitial mechanisms in biaxially strained Si-Ge films. Vertical offsets represent composition effect at constant strain; slopes represent strain effect at constant composition. Data from Kuo et al. [13]: circles: B in pure $\mathrm{Si}$; squares: B in SigoGe10; diamonds: $B$ in $\mathrm{Si}_{80} \mathrm{Ge}_{20}$. Straight lines determined from Eq. (2) using: (a) $\Delta V^{\text {relax }}$ for $\langle 110\rangle$ dumbbell interstitial in $\mathrm{Si}$ self diffusion from Tang et al. [15]; (b) the present preliminary data for the activation volume for B in Si; (c) $Q^{\prime}$ reported for OED of Si/Ge multilayers from Cowern et al. [14]; (d) $\Delta V^{*}$ for self diffusion by tetrahedral interstitial saddle point, from Antonelli and Bernholc [12]; (e) $\Delta V^{*}$ for self diffusion by bond-centered interstitial saddle point, from Antonelli and Bernholc. Vertical offsets of all lines are arbitrary; only the slopes are significant.

isolate the effect of strain on the interstitial-based component. They reported a value of $\mathrm{Q}^{\prime}=-12 \pm$ $6 \mathrm{eV}$ per unit strain for the interstitial contribution to oxidation-enhanced diffusion (OED).

In Fig. 4 we use Eq. (2) to compare the measured and predicted strain-dependence of boron diffusion in biaxially strained Si-Ge. The comparisons are made assuming (1) the anisotropy in the migration strain $\Delta \mathrm{V}_{/ /}^{\mathrm{m}}-\Delta \mathrm{V}_{\perp}^{\mathrm{m}}=0$ in all cases except that of Cowern et al., where no such assumption is necessary; (2) relaxation and migration volumes for point defects involving boron are identical to those calculated for pure $\mathrm{Si}$ (or Ge in the case of Cowern et al.); this assumption is not necessary for the present experiment performed on boron diffusion in $\mathrm{Si}$; (3) $\Delta \mathrm{Vm}=0$ for the $<110>$ dumbbell of Tang et al. [13]; (4) the biaxial modulus is $\mathrm{Y}=180.5 \mathrm{GPa}$ independent of composition and temperature. Only the slopes of the curves are relevant - their vertical offsets are arbitrary. The slopes of the data of Kuo et al. [13] for B diffusion in Si89Ge11 and $\mathrm{Si} 79 \mathrm{Ge}_{21}$ (From their Fig. 2) fall within the range of the other values whereas the slope of their data for B diffusion in pure Si under tensile strain (From their Fig. 3) is opposite in sign. Qualitatively, our data and the alloy data of Kuo et al. are consistent with an interstitial-based mechanism whereas their pure Si data are not. It would be very valuable to have calculations and experiments to determine the missing parameters and permit a rigorous comparison. The missing parameters are the formation or relaxation volumes for B diffusion by the interstitialcy or kick-out mechanism at the saddle point of the migration path, and the anisotropy in the migration strain.

\section{SUMMARY}

Preliminary results of effect of pressure on boron diffusion in silicon indicate that pressure enhances B diffusion in $\mathrm{Si}$ at $850{ }^{\circ} \mathrm{C}$, characterized by an average activation volume of $-0.125 \pm 0.02$ times the atomic volume. This result is qualitatively consistent with an interstitialbased diffusion mechanism. The results are also qualitatively consistent with several different atomistic calculations for $\mathrm{Si}$ self diffusion by interstitial-based mechanisms, with Cowern et al.'s interpretation of oxidation-enhanced diffusion in strained Si-Ge alloys, and with Kuo et al.'s 
results for B diffusion in biaxially strained Si-Ge alloys; these are all inconsistent with Kuo et al.'s results for B diffusion in biaxially strained pure Si films.

This research was supported by NSF-DMR-95-25907. Work at LANL was supported by the Division of Materials Sciences, DOE under contract W-7405-ENG-36 with the University of California. The authors are grateful to D.J. Eaglesham for providing the starting sample and to H.J. Gossmann for communication of his simulation results for comparison to the $\sigma^{2}$ results used here, for the performance of the Ar ambient anneal on this sample, and for much helpful discussion.

\section{REFERENCES}

${ }^{1}$ P.M. Fahey, P.B. Griffin and J.D. Plummer, Rev. Mod. Phys. 61, 289 (1989).

${ }^{2}$ H.-J. Gossmann, in Delta Doping of Semiconductors, edited by E.F. Schubert (Cambridge University Press, Cambridge, U.K., 1996), p. 253.

${ }^{3}$ M.J. Aziz, submitted to Appl. Phys. Lett.

${ }^{4}$ P.A. Stolk, D.J. Eaglesham, H.-J. Gossmann and J.M. Poate, Appl. Phys. Lett. 66, 1370 (1994).

${ }^{5}$ S. Mitha, M.J. Aziz, D. Schiferl and D.B. Poker, Appl. Phys. Lett. 69, 922 (1996).

${ }^{6}$ N.J. Hess and D. Schiferl, J. Appl. Phys. 71, 2082 (1992).

${ }^{7}$ Y.C. Zhao, W.B. Carter, S.D. Theiss, S. Mitha, M.J. Aziz and D. Schiferl, (unpublished).

${ }^{8}$ D.T. Wu, in Crucial Issues in Semiconductor Materials and Processing Technologies, edited by S. Coffa, F. Priolo, E. Rimini and J.M. Poate (Kluwer, London, 1992), p. 403.

${ }^{9}$ H.-J. Gossmann (private communication). The Bell Labs anneal took place in a furnace in argon of $99.95 \%$ purity flowing at 1.5 liters/minute at $850{ }^{\circ} \mathrm{C}$ for 45 minutes. The temperature was calibrated using solid phase epitaxial growth rates and using a thermocouple attached to the silicon and is believed to be accurate to within $\pm 10^{\circ}$. The furnace and procedure are identical to those reported in H.-J. Gossmann, C.S. Rafferty, F.C. Unterwald and T. Boone, Appl. Phys. Lett. 67, 1558 (1995).

${ }^{10}$ R.B. Fair, in Impurity Doping Process in Silicon, edited by F.F.Y. Wang (North-Holland, Amsterdam, 1981), Chapter 7.

${ }^{11}$ U. Södervall, M. Friesel and A. Lodding, J. Chem. Soc. Faraday Trans. 86, 1293 (1990).

${ }^{12}$ A. Antonelli and J. Bernholc, Phys. Rev. B 40, 10643 (1989).

${ }^{13}$ P. Kuo, J.L. Hoyt, J.F. Gibbons, J.E. Turner and D. Lefforge, Appl. Phys. Lett. 66, 580 (1995).

${ }^{14}$ N.E.B. Cowern, W.J. Kersten, R.C.M. de Kruif, J.G.M. van Berkum, W.B. de Boer, D.J. Gravesteijn and C.W.T. Bulle-Liewma, in Proc. 4th Int. Symp. on Process Physics and Modeling in Semiconductor Devices, eds. G.R. Srinivasan, C.S. Murthy, and S.T. Dunham, Electrochem. Soc. Proc. Vol. 96-4 (Electrochem. Soc., Pennington, NJ, 1996).

${ }^{15}$ M. Tang, L. Colombo, J. Zhu and T. Diaz de la Rubia, submitted to Phys. Rev. B. 